\title{
LAS COMPETENCIAS PROFESIONALES DEL TUTOR VIRTUAL ANTE LAS TECNOLOGÍAS EMERGENTES DE LA SOCIEDAD DEL CONOCIMIENTO
}

\section{THE VIRTUAL TUTOR COMPETENCES OF EMERGING TECHNOLOGIES TO THE KNOWLEDGE SOCIETY}

\author{
Cristina Sánchez Romero; csanchez@edu.uned.es \\ UNED. Facultad de Educación.
}

\begin{abstract}
Almudena Castellanos Sánchez; almudena.castellanos@unir.net
Universidad Internacional de La Rioja
\end{abstract}

\section{RESUMEN}

La investigación que presentamos tiene el objetivo de analizar las competencias profesionales del tutor virtual necesarias para desenvolverse con las tecnologías emergentes en espacios de formación on-line. La metodología de investigación de carácter descriptivo desarrollada con un cuestionario en formato digital recoge las habilidades y destrezas que se esperan de este grupo profesional. Entre los resultados destaca la necesidad de planificación formativa que capacite al tutor en el manejo y aprovechamiento didáctico de las mismas.

PALABRAS CLAVE: Educación a distancia, competencias, entornos virtuales de aprendizaje, tutor virtual, tutoría online.

\section{ABSTRACT}

The investigation that we sense beforehand has the aim to analyze the professional competitions that the virtual tutor needs to use emergent technologies in spaces of on-line formation. The methodology of investigation that has been in use is of descriptive character and a questionnaire has developed in digital format that summarizes the skills that a virtual tutor must have. The most important results are the need of formative planning in order that the virtual tutor could use in a didactic way the technologies.

KEYWORDS: Online learning, skills, virtual environments, virtual teacher, online tutoring. 


\section{INTRODUCCIÓN}

Los escenarios sociales, caracterizados principalmente por el adelanto imparable de la tecnología, la expansión de Internet, la globalización, el exceso de información, la fugacidad de los saberes y la importancia del conocimiento como generador de riqueza (Adell, 1997; OCDE, 1996; Cardona, 2002; Area, 2002; Sevillano, 2003; Cabero, 2007), reclaman cambios sobresalientes en el panorama educativo:

- Sistemas formativos preparados para educar a lo largo de toda la vida.

- Programas educativos que fomenten la capacidad de razonar, la de aprender cosas nuevas y el gusto por el estudio.

- Reconocimiento de las competencias digitales como factor clave de competitividad.

- Metodologías que conviertan al alumno en el protagonista de un aprendizaje reflexivo y colaborativo.

- Nuevos roles para los docentes, ahora mediadores del saber.

Una posibilidad para atender de manera integral a todas estas necesidades son los entornos virtuales de aprendizaje (Sigales, 2001; Medina, Domínguez y Sánchez, 2011), pues entre otras cosas, proporcionan una formación de calidad a una extensa diversidad de personas independientemente de la edad que tengan, dónde residan..., haciendo posible la educación a lo largo de toda la vida.

Todas estas transformaciones propias de la sociedad del conocimiento están afectando del mismo modo a la formación online, los adelantos conseguidos en el ámbito de las tecnologías están dando un giro sin precedentes a la oferta de la educación a distancia (Dondi, 2007; Castañeda, 2008; Johnson, 2010; Hanna, 2002). Una comunicación cada vez más bidireccional, la posibilidad de trabajar de forma colaborativa o la incorporación de los servicios que ofrece la Web 2.0, son sólo algunas de las posibilidades que hoy por hoy nos brindan los entornos virtuales.

Las nuevas plataformas online unifican lo mejor de la formación a distancia, individualizada, personalizada, flexible, cómoda, económica,... con el éxito de la formación presencial, la calidez humana. La oportunidad de ver, escuchar e interaccionar con el tutor en directo y a diario, así como con los compañeros de curso, incrementa notablemente la sensación de pertenencia y acaba con el aislamiento y la desorientación que a veces tiende a darse en la formación online. Tecnologías como el video-streaming o la televisión sobre protocolos IP (IPTV), están contribuyendo cada vez más a ello.

Pero los entornos virtuales del siglo XXI ¿exigen nuevas competencias para los tutores que realizan su labor online?, y en caso afirmativo, ¿cuáles son?

Si bien son muchos los autores que han analizado el nuevo perfil del tutor virtual, tal es el caso de Marcelo (2002, 2005), Barker (2002), Salmon (2004), Gros y Adrián (2004), Casati y Casati (2005), Llorente (2005), Bautista (2006), Vásques (2007), Bosom (2007), Medina, Medina y Sánchez (2007), Ortega (2007), Coll y Monereo (2008), Pagano (2008), García Aretio (2009) o más recientemente Area, Gutiérrez y Vidal (2012), llegando a hablar de una realfabetización digital compensatoria que incluya la dimensión instrumental, cognitiva- 
intelectual, sociocomuniaccional, axiológica y emocional (Area, Gutiérrez y Vidal, 2012, p.30), se han encontrado pocos estudios dedicados a estudiar las competencias reales de los tutores e-learning en la era digital. Hoy por hoy se espera del tutor virtual que sepa utilizar la Red como la biblioteca universal que es, que sea un trabajador consciente de sus derechos y responsabilidades en el nuevo Internet de los servicios, que domine las formas hipertextuales de organización de la información en la Red, que sea capaz de desarrollar comportamientos sociales basados en la colaboración dentro del espacio público de Internet, que sea un consumidor crítico de los productos audiovisuales, que produzca y publique ideas a través de los nuevos entornos digitales o que disponga de las habilidades necesarias para interaccionar en entornos virtuales tridimensionales de realidad inmersiva, simulada y aumentada (Area, Gutiérrez y Vidal, 2012, p.31).

El proyecto Escuela de Nuevas Tecnologías de EducaRed (2006) promovido por Fundación Telefónica, ofrece a distancia cursos para la formación del profesorado en materia tecnológica con el objetivo de mejorar la calidad de enseñanza. EducaRed, como referente de innovación para los docentes, pretende para cursos futuros, ofrecer una metodología formativa mejorada, enriquecida con todos los adelantos tecnológicos más punteros dándoles así la oportunidad a los alumnos que se matriculan en sus cursos, de poder aprender en un entorno de trabajo que integra todas esas herramientas que empiezan a estudiar, blogs, wikis, etc. ¿Cuentan los tutores virtuales educared con las competencias necesarias para desenvolverse con éxito en estos escenarios reformados?

\section{OBJETIVOS}

Nuestra investigación considera que los resultados del proyecto están relacionados con la labor de tutorización de los profesionales que lo desarrollan. Por ello, los objetivos fueron:

- Analizar si las competencias con las que actualmente cuentan los profesores virtuales, son suficientes para desarrollar su actividad en un entorno mejorado, enriquecido con los avances tecnológicos.

- Desarrollar un plan de formación que capacite a los tutores virtuales para para el manejo de un entorno de enseñanza virtual enriquecido con la tecnología más puntera.

Al mismo tiempo que la misma, puede resultar interesante para otros profesionales del ámbito educativo que estén decididos a implementar entornos virtuales con tecnología avanzada, pues conocerán de primera mano las competencias necesarias para trabajar en ellos y podrán extrapolar los resultados de este estudio a poblaciones similares.

\section{METODOLOGÍA}

Para cuantificar de forma objetiva las competencias de este grupo de profesionales se ha empleado un cuestionario anónimo que cumplimentaron en formato digital; la elección de este instrumento viene dada por el propio problema de investigación, pues si lo analizamos desde un punto de vista cronológico (Fox, 1981), la respuesta al problema está claramente en el presente, por lo que se ha utilizado un enfoque descriptivo con el que hemos intentado 
llegar a una comprensión más profunda de las condiciones actuales de este grupo de profesionales. Hemos optado por una versión digital del cuestionario teniendo en cuenta las recomendaciones de algunos autores (Ballester, 2001; García López, 1999), dada su versatilidad para adaptarse a las necesidades de la investigación, la rapidez a la hora de recabar datos, el bajo coste que tiene al desarrollarse de manera online, su capacidad para llegar de manera efectiva a la población seleccionada, los escasos sesgos que origina o la baja resistencia que ofrece a los encuestados por lo novedoso que resulta.

En definitiva, está constituido por 18 preguntas que nos han permitido obtener información directa sobre las competencias que hoy por hoy nos ofrece la Era Digital.

La muestra seleccionada fueron 113 profesionales que forman parte de la plantilla de tutores del programa Escuelas de Nuevas Tecnologías EducaRed,

Con la finalidad de desarrollar, en caso necesario, un plan de formación que capacite a los mismos o a los que en un futuro pretendan formar parte de la plantilla.

La organización del cuestionario en siete secciones diferentes, nos facilitó recabar datos de acuerdo con un orden temático establecido.

Esta disposición nos ha ayudado a evitar preguntas innecesarias y a cuidar la relación de éstas con el objetivo de la investigación. Las secciones se ordenaron de tal forma que despertasen el interés del informante, de lo más sencillo a lo más complejo (Hernández Rodríguez, 2004). Para impedir que los tutores rehusaran responderlo, las preguntas están planteadas de manera clara, precisa y sencilla, en orden a esas 7 diferentes dimensiones mencionadas. Se trata de un cuestionario anónimo, con el fin de no condicionar las respuestas. De la misma forma, para evitar que la persona que da las instrucciones para cumplimentar el cuestionario influyera con su actitud en las respuestas, todos los tutores virtuales han recibido las mismas instrucciones por escrito. El hecho de que hayan podido hacerlo a través de Internet, tiene como ventaja que se pueda aplicar el cuestionario a una muestra mayor de tutores independientemente del País o la Comunidad Autónoma de procedencia, pues el investigador no necesita estar presente. No obstante, con esta metodología corríamos el riesgo de que algunos tutores no nos enviaran respuesta. En este caso, acordamos que si el porcentaje era menor al $80 \%$ de respuestas, haríamos un segundo envío con el mismo cuestionario a aquellas personas que no lo hubieran mandado.

Por otro lado, se ha procurado que todas las preguntas y respuestas cumplieran con las características propuestas por algunos autores (Fox, 1981), claridad en el lenguaje, concreción en cuanto a contenidos y tiempos, unicidad en el propósito, independencia de otros supuestos y posibles sugerencias, coherencia lingüística y gramatical y suficientes opciones de respuesta. Aprovechando su condición digital, decidimos que las preguntas de mayor interés para la investigación, fueran de carácter obligatorio de tal forma que el participante no pudiera avanzar si no eran respondidas.

Para contrarrestar los inconvenientes que supone emplear un cuestionario impersonal (Fox, 1981):

- Se limitó al máximo la extensión del cuestionario, se ha procurado incorporar exclusivamente las preguntas críticas para la investigación. 
- Se seleccionó predominantemente un modelo de respuesta estructurada.

- Se redactó una introducción clara, sincera.

- Se avisó que se harían públicos los resultados.

A la hora de seleccionar una herramienta que nos permitiera crear un cuestionario digital, se optó por el gestor personalizado LimeSurvey que utilizamos habitualmente desde el portal EducaRed para medir la opinión de los usuarios. Se trata de una aplicación libre -open source- desarrollada en tecnología PHP y personalizada para Fundación Telefónica que utiliza una base datos MySQL y cuyo objetivo es que cualquier usuario sin conocimientos en programación informática, sea capaz de publicar y analizar cuestionarios. Las razones de utilización de esta herramienta, fueron principalmente la fiabilidad en el registro de datos que hace de cada cuestionario y la facilidad de cara a estandarizar la información recogida.

En cuanto a la necesidad de validación de la herramienta, se sometió al juicio de quince expertos para que analizaran la adecuación de éste a la investigación, la compresión de los ítems y la coherencia interna, a través de una escala Likert de 5 puntos ("muy en desacuerdo", "en desacuerdo", "de acuerdo", "bastante de acuerdo", "totalmente de acuerdo"). El valor más seleccionado para estos tres ítems fue el equivalente a totalmente de acuerdo (5).Su consistencia interna fue de 0.86 en el Alfa de Cronbach.

Una vez diseñado nuestro cuestionario, el gestor nos devolvió la siguiente dirección URL preparada para ser enviada a los participantes del estudio. No obstante, antes de comenzar su difusión se probó para descartar posibles errores técnicos y de usabilidad. Un grupo piloto formado por 4 personas con un perfil similar al de la muestra, sirvieron para hacer las pruebas. Desde este enlace se puede ver el cuestionario en formato digital: http://encuestas.telefonicalearningservices.com/encuestas/index.php?sid=54642\&lang=es

En una primera fase, a través de la plataforma de correo interno que empleamos para nuestro trabajo, se envió a los profesores la dirección URL a través de la cual pudieron realizar su cuestionario anónimo y se les explicó por escrito las condiciones de realización de éste. A la semana siguiente se volvió a enviar un nuevo correo electrónico a aquellos profesionales que no habían rellenado el cuestionario con el fin de animarles a hacerlo. Transcurridas 2 semanas, el sistema de gestión LimeSurvey detecta 103 envíos completos. Como este número de cuestionarios entraba dentro de los límites previsibles - algo más del $91 \%$-, dimos por terminada esta fase y pasamos al análisis de los datos.

\section{RESULTADOS Y DISCUSIÓN}

En primer lugar diremos que los datos han sido analizados con la ayuda del gestor LimeSurvey, que cuenta con funcionalidades básicas de análisis estadístico. El modo en que se presentaron los resultados se basó fundamentalmente en gráficas circulares.

\subsection{Aspectos Generales}

De los 103 cuestionarios recibidos 64 pertenecen a mujeres, consiguiendo así un porcentaje mayor de respuesta $62 \%$, frente al $38 \%$ de los hombres. Según los datos recogidos, predominan los tutores que pertenecen a la franja de edad que va de los 31 a los 40 años (54 
sujetos de los 103 encuestados), siendo minoritarios los que tienen de 41 a 50 años (10 sujetos). La franja de los 20 a los 30 años ha sido marcada por un $38 \%$ de los tutores (39 sujetos). Es destacable que ninguno de los tutores que han respondido al cuestionario tiene más de 50 años.

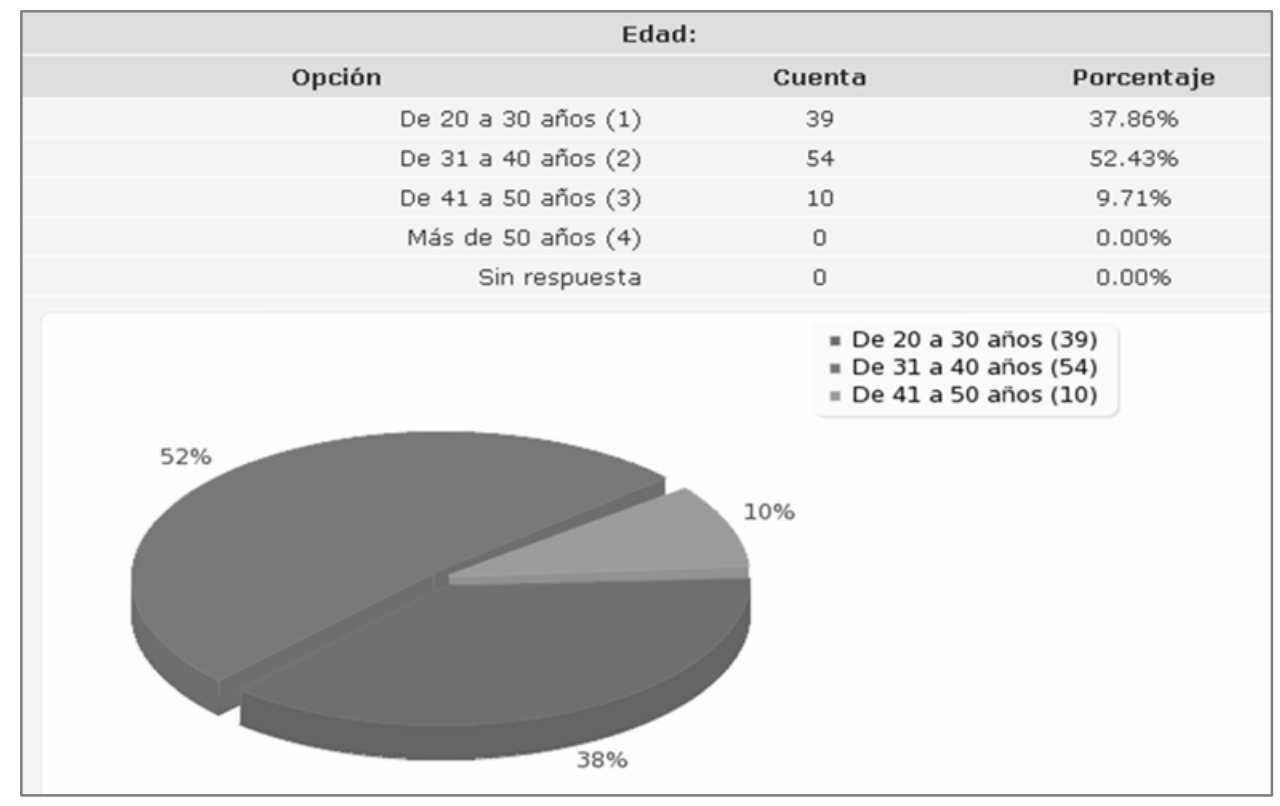

Gráfico 1. Distribución por edades de los tutores virtuales

A la pregunta de si utilizan habitualmente tecnologías de la información y la comunicación, siendo la respuesta una escala del 1 al 5 donde 1 es Nada y 5 Mucho, el 78\% de los participantes responde que las utiliza Mucho. Ninguno de los participantes afirma no utilizar las tecnologías habitualmente dato que chocaría bastante al tratarse de tutores virtuales.

A pesar de que existen estudios que relacionan el buen desempeño como tutor online con haber tenido experiencias como alumno en línea (Vásquez, 2007), sólo un $11 \%$ de los encuestados afirma tener Mucha experiencia como alumno en línea. El $36 \%$ de los encuestados afirman tener Nada (9 tutores) o Poca experiencia (28 tutores). El 21\% contestan tener Bastante experiencia como alumno virtual. En cuanto a su experiencia como tutores online, sólo 3 de los 103 encuestados afirma no tener experiencia como tutor online. Entre los tutores que manifiestan tener experiencia como tutor online, el 39\% lleva menos de 2 años trabajando en esta profesión, el 34\% lleva de 2 a 4 años trabajando y el $27 \%$ lleva más de 4 años. 


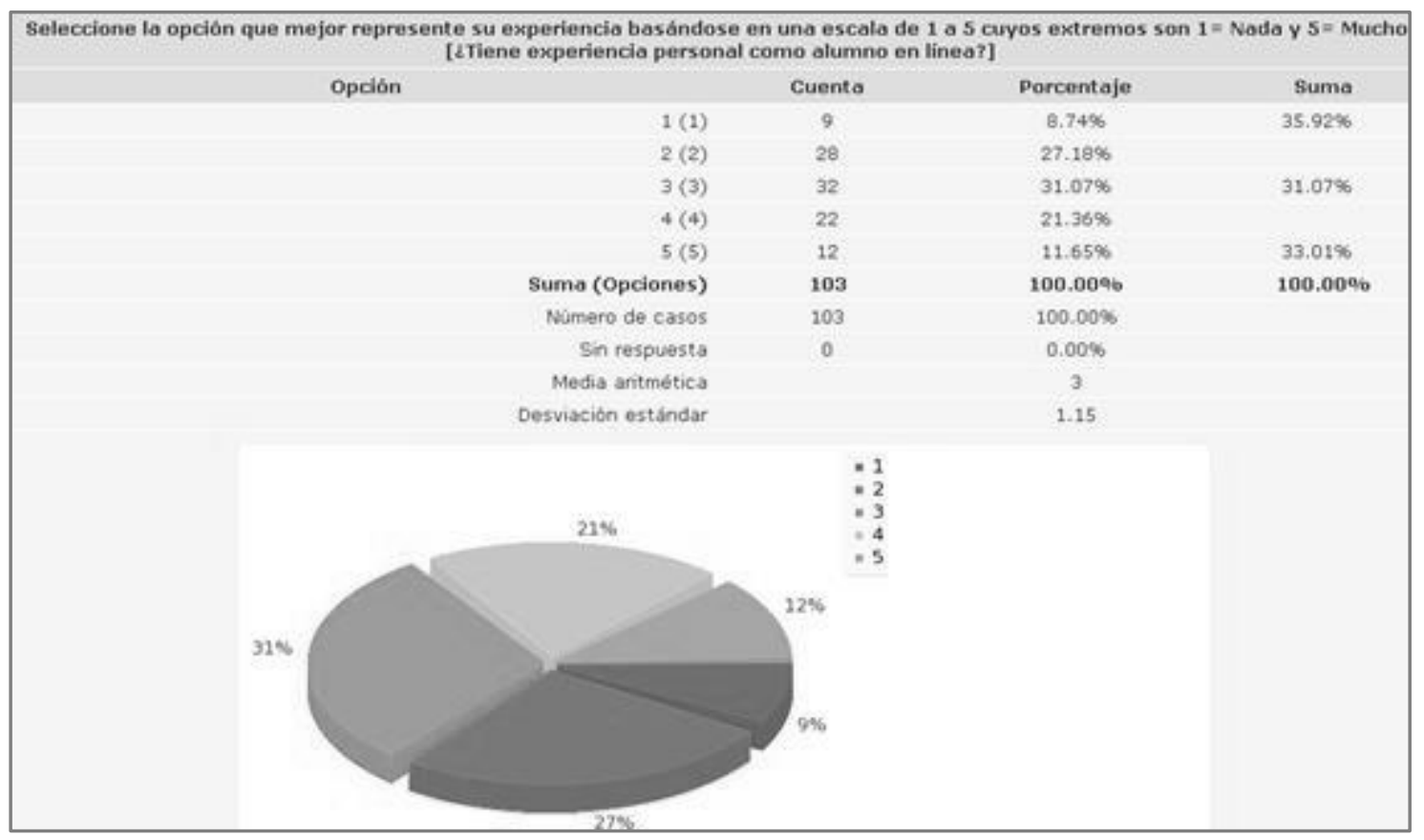

Gráfico 2. ¿Tiene experiencia personal como alumno en línea?

\subsection{Competencias Tecnológicas}

En una escala de 1 a 5 que mide los conocimientos, habilidades prácticas y actitudes para trabajar con Procesadores de Textos, donde 1 equivale a Mínimo Acuerdo y la puntuación 5 a Máximo Acuerdo, la inmensa mayoría de los encuestados mantiene tener las competencias necesarias para trabajar con estos programas. Un 73\% selecciona la máxima puntuación (5 puntos) y un $25 \%$ elije la puntuación 4 . Es destacable cómo ninguno de los participantes ha escogido la puntuación 1 ó 2 que confirmaría el escaso dominio de estas aplicaciones. Sólo 28 de los participantes (27\%) afirma tener las competencias necesarias para trabajar con Bases de Datos. El 35\% de los encuestados mantiene que no las domina. En cuanto a las Hojas de Cálculo, 46 de los 103 encuestados están completamente de acuerdo en que tienen competencias suficientes para trabajar con estos programas. Ningún encuestado mantiene no manejar las hojas de cálculo, no obstante 18 personas confirman tener un dominio Medio. El $98 \%$ de los encuestados considera estar perfectamente preparados para trabajar con Presentadores de Diapositivas. 


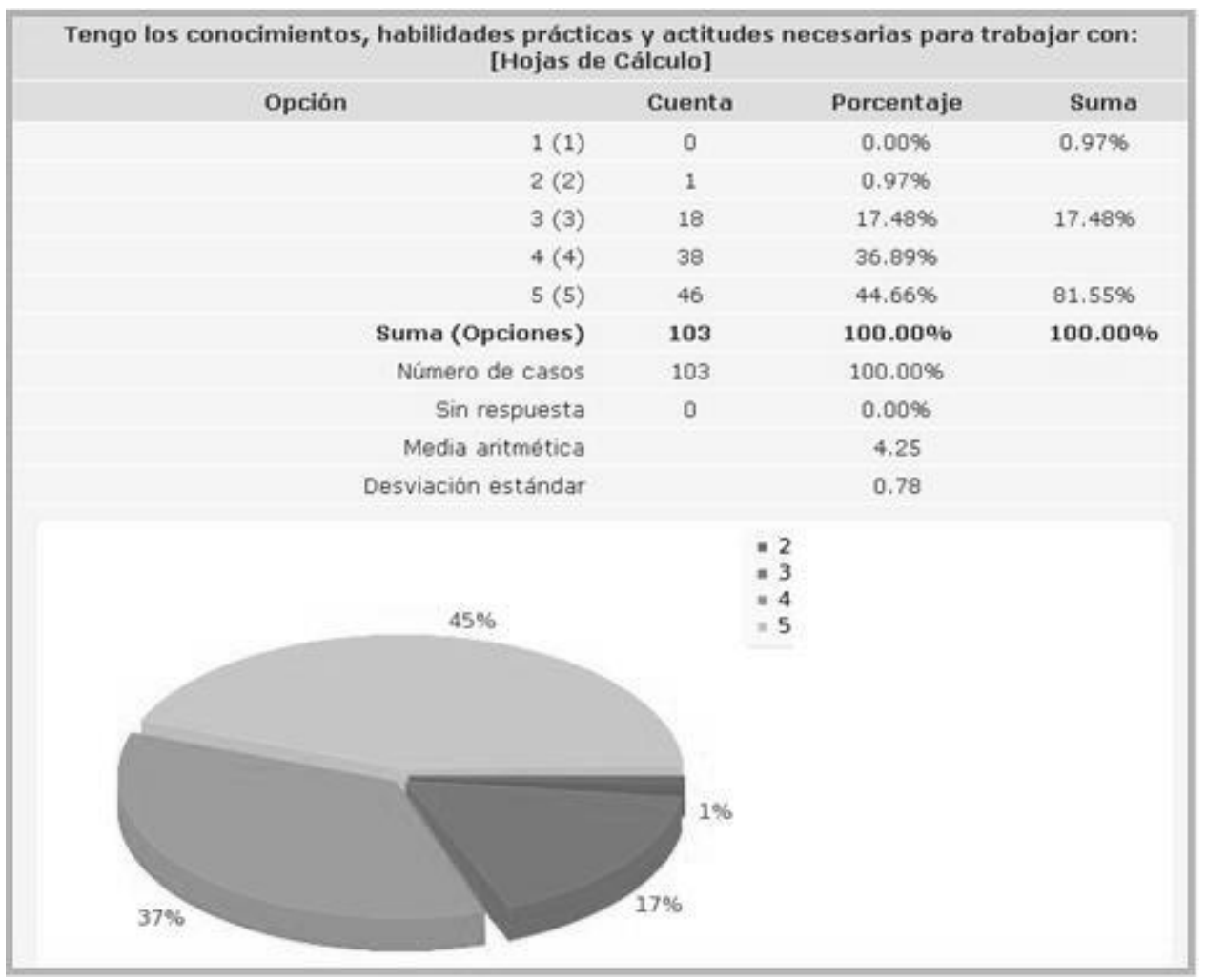

Gráfico 3. Nivel de Competencias para trabajar con Hojas de Cálculo

En una escala de 1 a 5 que mide los conocimientos, habilidades prácticas y actitudes para Diseñar Páginas Web, donde 1 equivale a Mínimo Acuerdo y la puntuación 5 a Máximo Acuerdo, el $50 \%$ de los participantes ha señalado que no está preparado para diseñar páginas web, sólo 24 personas de 103 consideran tener suficientes competencias. La mayoría de los encuestados (62\%) manifiestan que no dominan los Programas de Diseño gráfico. Sólo 9 personas afirman tener las competencias necesarias para trabajar con programas para la Creación y Edición de Audio, frente al 55\% que confiesan no ser competente. La inmensa mayoría de los encuestados (98\%) afirma tener las competencias necesarias para trabajar con el Correo Electrónico. Sin embargo, únicamente 4 personas de las 103, afirman manejar perfectamente la herramienta Blog. En cuanto a programas para la Transferencia de Archivos, sólo 3 personas confirman tener las competencias necesarias. El $55 \%$ de los encuestados confirman que no tienen competencia para trabajar con Marcadores Sociales. Respecto a los programas para Editar Imágenes, el $18 \%$ de los encuestados afirma contar con las competencias necesarias para trabajar con estas aplicaciones. El $64 \%$ de los encuestados reconoce que no es competente en lo que se refiere a Programas para la Creación de 3D. A la hora de Diseñar actividades y pruebas de evaluación en formato digital, el $37 \%$ de los encuestados manifiesta que no es competente, sólo un $17 \%$ afirma estar capacitado. El 59\% de los encuestados confiesa que no tiene competencias a la hora de manejar programas de Screencast, sólo el $9 \%$ de los tutores afirma tenerla. En cuanto al manejo de las Videoconferencias, el $37 \%$ de los preguntados confiesa que no tiene competencia. Aplicaciones tan útiles como son las que permiten compartir en tiempo real un Escritorio remoto, tan solo saben manejarlas el $2 \%$ de los encuestados. El $43 \%$ de los encuestados reconoce no ser competente a la hora de Editar Video. El $88 \%$ de los encuestados afirma tener el grado máximo de competencias a la hora de trabajar con la herramienta de comunicación Foro y el $94 \%$ maneja a la perfección los Chats. Servicios más 
novedosos como son los Wikis exclusivamente los manejan un $11 \%$ de los tutores consultados. En cuanto a las Redes Sociales, el 22\% de los participantes dice no tener competencia y un $53 \%$ dice tener competencias medias.

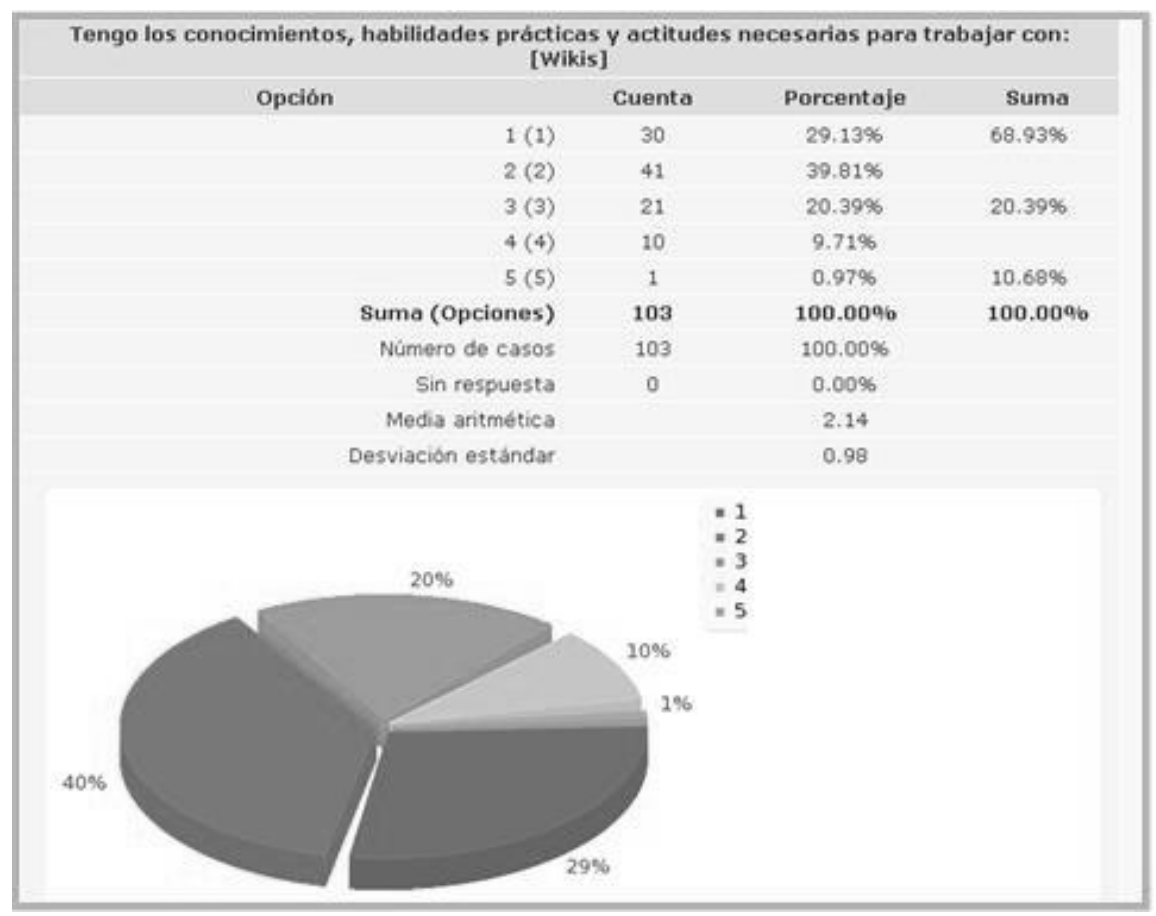

Grafico 4. Nivel de Competencias para trabajar con Wikis

\subsection{Competencias de Diseño Pedagógico, Tutoriales y de Relación Empática}

En una escala de 1 a 5 que mide los conocimientos, habilidades prácticas y actitudes para Trabajar con los principios didácticos y las principales teorías de aprendizaje, donde 1 equivale a Mínimo Acuerdo y la puntuación 5 a Máximo Acuerdo, el 92\% de los encuestados manifiesta tener una adecuada competencia. A la hora de Diagnosticar las necesidades de formación en función de la demanda existente en el mercado, el $88 \%$ de los encuestados dice tener suficiente competencia. El 93\% de los encuestados dice ser competente a la hora de Trabajar de manera coordinada con un equipo interdisciplinar.

A la hora de Seleccionar el sistema de tutoría más adecuado a cada momento, el $86 \%$ de los participantes reconoce tener una adecuada competencia en este sentido. El $85 \%$ de los tutores dice tener competencia en cuanto a Actuar como facilitador de recursos y de información útil para los alumnos. Respecto a la tarea de Moderar debates en foros y chats y Retroalimentar las discusiones, el $91 \%$ de los preguntados dice tener una adecuada competencia. El $85 \%$ de los encuestados afirma que tiene competencia para Detectar necesidades y expectativas de los alumnos así como estados de soledad y aislamiento. Baja el número de participantes (42\%) que se encuentran capacitados para Fomentar la Metacognición en el alumno. El 92\% de los tutores reconoce estar muy capacitado para Mantener un estilo de comunicación no autoritario, motivador, amistoso. La gran mayoría (91\%) de los tutores consideran que tienen un nivel adecuado de competencias a la hora de Escuchar y Dar Consejos. Por último, el $87 \%$ de los participantes considera que tiene competencias a la hora de Crear un Clima de Confianza para que el alumno pueda expresar sus problemas. 


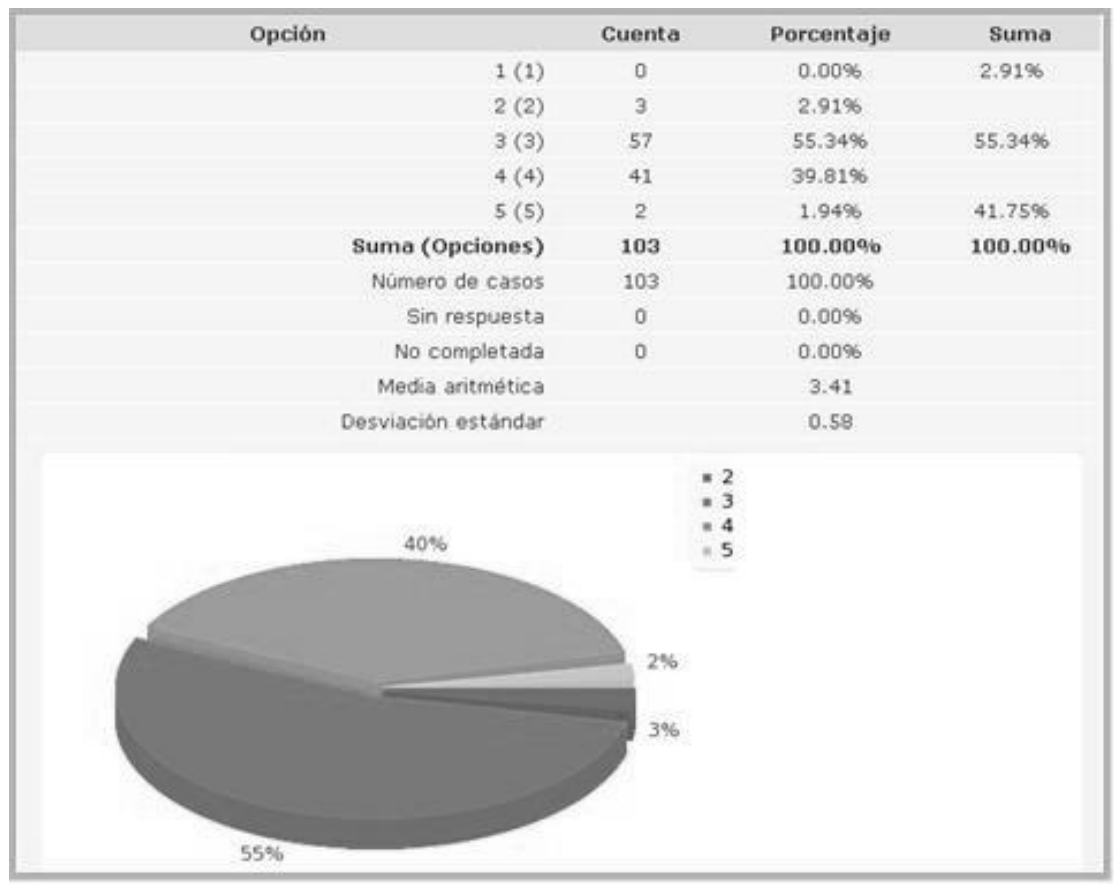

Gráfico 5. Competencias a la hora de fomentar la Metacognición

\subsection{Discusión.}

Procedemos a discutir los resultados en función de los objetivos que nos hemos planteado en la investigación.

Respecto a nuestro objetivo general "Analizar si las competencias con las que actualmente cuentan los profesores virtuales, son suficientes para desarrollar su actividad en un entorno mejorado tecnológicamente", los resultados muestran cómo los tutores dominan principalmente las diferentes aplicaciones de los paquetes de ofimática, sobretodo los Procesadores de Textos y no tanto las Bases de Datos, pues consideramos que se trata de programas menos extendidos fuera del ámbito profesional como hemos visto en la base teórica de esta investigación.

Esta clara ventaja que le lleva la ofimática a otro tipo de aplicaciones como el Diseño de páginas web o el Diseño gráfico, probablemente se deba a lo generalizado que está el uso de estas aplicaciones en la vida diaria, además de la apuesta de los planes de formación continua para educadores por herramientas básicas como Word, PowerPoint o Excel, hay que recordar que como dice Marcelo (2002) son destrezas básicas que se esperan de un tutor el manejo de este tipo de software.

Por otro lado, aunque no les faltan competencias a la hora de trabajar con herramientas para la comunicación como el Correo Electrónico, los Foros o los Chats, servicios que desde ya algunos años vienen ofreciendo las plataformas de aprendizaje online y cuyo manejo se contempla como competencia para muchos autores (Marcelo 2002; Barker 2002; Salmon, 2004), no ocurre lo mismo con herramientas más novedosas como la Videoconferencia o las Redes Sociales, lo que nos lleva a afirmar que sería necesario una formación continua en herramientas de comunicación más recientes. 
En cuanto a nuestro objetivo específico de conocer las competencias del tutor para trabajar con programas que podrían ayudarle a que desarrollara contenidos digitales más novedosos como es la creación y edición de audio o la edición de imágenes, probablemente requieran de una formación complementaria si queremos disponer de un material didáctico más interactivo, amigable, a la carta (Marcelo, 2005).

A lo largo de este trabajo hemos hablado de tecnologías que han dejado de ser incipientes para instalarse con fuerza en el ámbito educativo y con mucho argumento en la educación a distancia, tal es el caso de los Wikis, las Redes Sociales, los Blogs, los programas para compartir Escritorio en Tiempo Real o los Marcadores Sociales, los bajos resultados demuestran la necesidad de un acercamiento de los tutores a estos nuevos servicios 2.0 y a sus implicaciones pedagógicas.

En lo que se refiere al objetivo de medir las Competencias de Diseño Pedagógico de un tutor virtual, por lo general el grupo de tutores parece tener los conocimientos, las habilidades prácticas y las actitudes necesarias para trabajar con las principales teorías del aprendizaje, diagnosticar necesidades de formación, trabajar en equipos interdisciplinares... Afirmamos por tanto que no sería necesario llevar a cabo una acción formativa complementaria en este sentido.

Respecto al objetivo de analizar las Competencias Tutoriales, los resultados ponen de manifiesto lo capacitado que está el equipo de tutores en este sentido por lo que no sería preciso llevar a cabo acciones formativas complementarias en esta materia.

En lo que se refiere al objetivo de analizar las Competencias Evaluativas, si bien la encuesta demuestra que tienen un gran dominio de las principales actividades, evaluar desde un enfoque formativo y continuo, evaluar el trabajo de uno mismo como tutor, marcar metas realistas ... los tutores parecen tener más dificultades a la hora de fomentar la metacognición, atender mediante la evaluación a las diferencias de los alumnos o emplear la evaluación como proceso de comunicación lo que exige de formación continua en este sentido.

De igual forma nos marcamos el objetivo de analizar las Competencias de Gestión y Organización de cursos online en los tutores virtuales, en este caso y aunque en principio no entran dentro de las funciones de estos profesionales en EducaRed, algunos de los tutores dominan muchos de los procesos debido probablemente a experiencias laborales anteriores. Demostradas las carencias en algunas tareas, como publicitar un curso, poner en práctica estándares internacionales o buscar financiación para un curso, sería conveniente formar a este grupo de profesionales en Gestión y Organización de Acciones Formativas para un mejor reciclaje laboral.

Por último, en cuanto al objetivo de analizar las Competencias de Comunicación y Relación Empática, los niveles más bajos los encontramos en las competencias que tienen que ver con emplear el humor en línea, plantear negociaciones o fomentar el intercambio entre los alumnos; mientras que las puntuaciones más altas las tienen expresarse verbalmente con claridad, mantener un estilo de comunicación no autoritario, expresarse por escrito con claridad o ponerse en el lugar del alumno, lo que nos lleva a pensar que sería necesario que recibieran formación en este sentido, lo que también confirman estudios como los de Casati y Casati (2005). 
Otro aspecto de interés para este estudio han sido las cualidades personales que predominan en los tutores, destacando principalmente la capacidad de escucha.

\section{CONCLUSIONES}

En la investigación los resultados obtenidos nos confirman que los profesionales analizados donde predomina el género femenino y con edades comprendidas entre los 20 y 50 años, se puede afirmar que utilizan las tecnologías de la información y la comunicación habitualmente, condición que puede ayudarnos en el caso de tener que formarles a través de Internet $o$ actualizarles ante las nuevas demandas.

Un aspecto a destacar es la escasa experiencia que tienen como alumnos online, solamente el $33 \%$ de los encuestados dice tener experiencia, lo que apunta ya una posible carencia vivencial, como se ha explicado en el trabajo, existen estudios que aseguran que tener un buen bagaje como alumno online contribuye a que uno sea un excelente tutor virtual.

En contraposición, el $97 \%$ de los participantes en el estudio tienen experiencia como tutores online, incluso la mayoría (59\%) lleva trabajando más de dos años en esta profesión.

Sin ir más lejos, algunas carencias en el plano tecnológico, sobre todo en lo que se refiere a los nuevos servicios que ofrece la Web 2.0 y herramientas avanzadas para la creación de contenido, podrían abordarse mediante acciones formativas con metodología online, de tal forma que a la vez que se reciclan, los tutores amplían sus experiencias como alumnos online, algo escasas tal y como demuestra el estudio. En este sentido los resultados coinciden con los estudios que mantienen que si bien los tutores demuestran una alta competencia psicológica, pedagógica y comunicativa, no ocurre lo mismo con sus aptitudes más técnicas exceptuando las herramientas más extendidas como son el chat, los foros y el correo electrónico (Urdaneta y Guanipa, 2007).

Por ello proponemos una formación didáctico-tecnológica acorde al panorama actual, clave para convertirnos en una Sociedad de la Innovación. La cultura digital exige del profesor elearning una realfabetización compensatoria que le haga consciente de cómo inciden las tecnologías en el entorno cultural y político de nuestra sociedad, del conjunto de sentimientos que provoca la experiencia en entornos virtuales, una realfabetización digital que capacite al docente online para el dominio técnico de la tecnología propia de su tiempo, que le permita utilizar de forma inteligente la información que tiene ahora a su alcance, analizarla críticamente y reconstruirla y establecer comunicaciones fluidas con las personas a través de las tecnologías, desarrollando normas de comportamiento que impliquen una actitud social positiva hacia los demás -colaboración, empatía, respeto...- (Area, Gutiérrez y Vidal, 2012, p.30).

Los profesionales que actualmente forman parte del proyecto Escuela de Nuevas Tecnologías EducaRed y que para los años consecutivos comenzarán a trabajar en una nueva plataforma enriquecida con los adelantos tecnológicos más punteros, necesitan un plan de formación interna que les capacite para su nuevo desempeño, este análisis servirá de guía para el desarrollo del mismo. A su vez, las necesidades aquí recogidas bien podrían servir para diseñar planes de formación continua para este sector profesional en general. 


\section{REFERENCIAS}

ADELL, J. (1997). Tendencias en educación en la sociedad de las tecnologías de la información. EDUTEC, 7 [en línea]. Disponible en: http://www.uib.es/depart/gte/revelec7.html [consulta: 2012, 7 de noviembre]

AREA, M. (2002). Problemas y Retos educativos ante las tecnologías digitales en la sociedad de la información. Quaderns Digitals, 28 [en línea]. Disponible en: http://www.quadernsdigitals.net/index.php?accionMenu=hemeroteca.VisualizaArtic ulolU.visualiza\&articulo id=6351 [consulta: 2012,7 de noviembre]

AREA, M.; GUTIÉRREZ, A. y VIDAL, F. (2012). Alfabetización digital y competencias informacionales. Barcelona: Ariel

BALLESTER, L. (2001). Bases Metodológicas de la Investigación Educativa. Palma: Universidad de las Islas Baleares.

BARKER, P. (2002). Skill Sets for Online Teaching [en línea]. http://www.eric.ed.gov/ERICDocs/data/ericdocs2sql/content storage 01/0000019b /80/1b/17/4f.pdf [consulta: 2012,7 de noviembre]

BAUTISTA, G. (2006). Didáctica universitaria en entornos virtuales de enseñanza-aprendizaje. Madrid: Narcea

BOSOM, A. (2007). Online Tutor: An experience of network collaborative learning [en línea]. http://ceur-ws.org/Vol-186/07.pdf [consulta: 2012, 7 de noviembre]

CABERO, J. (2007). Nuevas Tecnologías Aplicadas a la Educación. Madrid: McGraw-Hill

CARDONA, G. (2002). Tendencias Educativas para el siglo XXI. Educación Virtual, Online y Elearning. Elementos para la discusión. Edutec: Revista electrónica de tecnología educativa, 15 [en línea]. Disponible en: http://edutec.rediris.es/Revelec2/revelec15/cardona.pdf [consulta: 2012, 7 de noviembre]

CASATI, S. y CASATI, N. (2005). Apuntes sobre el rol del tutor virtual [en línea]. En http://espacio.uned.es/fez/view.php?pid=bibliuned:19514 [consulta: 2012, 7 de noviembre]

CASTAÑEDA, L. (2008). Entre construir Entornos Virtuales de Enseñanza-Aprendizaje y enriquecer Entornos Personalizados de Aprendizaje [en línea]. Comunicación presentada al Congreso EDUTEC 2008: Las TIC Puente entre culturas. Iberoamérica y Europa. Santiago de Compostela, España. Septiembre 2008. Disponible en: http://www.lindacastaneda.com/publicaciones/castanedaedutec2008.pdf [consulta: 2012, 7 de noviembre]

COLL, C. y MONEREO, C. (2008). Psicología de la Educación Virtual. Madrid: Morata.

DONDI, C. (2007). The underground rivers of innovative e-Learning: a preview from the HELIOS Yearly Report 2006/07 [en línea]. Disponible en 
http://www.elearningeuropa.info/files/media/media12720.pdf [consulta: 2012, 7 de noviembre]

FOX, D. (1981). Investigación en Educación. Pamplona: Universidad de Navarra.

GARCÍA LÓPEZ, P.A. (1999). Problemas en el diseño y validación de cuestionarios. Estadística Española, 41 (144), 19-46.

GARCÍA ARETIO, L. (2009). ¿Por qué va ganando la educación a distancia? UNED: Madrid

GROS, B. y ADRIÁN M. (2004). Estudio sobre el uso de los foros virtuales para favorecer las actividades colaborativas en la enseñanza superior. Teoría de la Educación, 5.

HANNA, D.E. (2002). Nuevas perspectivas sobre el aprendizaje en la enseñanza universitaria. En Hanna, D.E. (ed). La enseñanza universitaria en la era digital. ¿Es ésta la universidad que queremos? Barcelona: Octaedro_EUB, 59-81.

HERNÁNDEZ RODRÍGUEZ, O. (2004).Estadística elemental para Ciencias Sociales. Costa Rica: Universidad de Costa Rica.

JOHNSON, L., LEVINE, A., y SMITH, R. (2010). Horizon Report. Austin, Texas: The New Media Consortium [en línea]. Disponible en: http://www.nmc.org/pdf/2010-HorizonReport-es.pdf [consulta: 2012, 7 de noviembre]

LLORENTE, M.C. (2006). El tutor en e-learning: aspectos a tener en cuenta. Revista Edutec, no 20, 1-24.

MARCELO, C. y OTROS (2002). E-learning teleform@ción. Diseño, desarrollo y evaluación de la formación a través de internet. Barcelona: Ediciones Gestión 2000.

MARCELO, C. (2005). Estudio sobre Competencias profesionales para e-learning [en línea]. Disponible en http://prometeo3.us.es/publico/images/competencias.pdf [consulta: 2012, 7 de noviembre]

MEDINA, A., MEDINA, J. A. y SÁNCHEZ, C. (2007). Las Tareas del Tutor en la Enseñanza Virtual: su aportación a la formación práctica de los estudiantes [en línea]. Disponible en: http://redaberta.usc.es/uvi/public html/images/pdf2007/antonio medina.pdf [consulta: 2012, 7 de noviembre]

OCDE (2005). La Definición y Selección de Competencias Clave [en línea]. Disponible en: http://www.deseco.admin.ch/bfs/deseco/en/index/03/02.parsys.78532.downloadLis t.94248.DownloadFile.tmp/2005.dscexecutivesummary.sp.pdf [consulta: 2012, 7 de noviembre]

ORTEGA, I. (2007). El Tutor virtual: aportaciones a los nuevos entornos de aprendizaje. Revista Teoría de la Educación, 2, 100-115.

PAGANO, C.M. (2008). Los tutores en la educación a distancia. Un aporte teórico. Revista de Universidad y Sociedad del Conocimiento, 2, 1-11.

SALMON, G. (2004). E-actividades: El Factor clave para una formación en línea activa. Editorial UOC: Barcelona. 
SEVILLANO, M. L. (2003). Nuevas Tecnologías aplicadas a la Educación. Madrid: UNED.

SIGALÉS, C. (2001). El potencial interactivo de los entornos virtuales de enseñanza y aprendizaje en la educación a distancia [en línea]. Disponible en: http://www.uoc.edu/web/esp/art/uoc/sigales0102/sigales0102.html [consulta: 2012, 7 de noviembre]

URDANETA, M y GUANIPA, M. (2007). Perfil de competencias del docente tutor en línea para la educación a distancia [en línea]. Revista Eduweb. http://servicio.bc.uc.edu.ve/educacion/eduweb/vol2n2/art6.pdf consulta: 2012, 20 de noviembre]

VÁSQUEZ, M. (2007). Tutor Virtual: Desarrollo de Competencias en la Sociedad del Conocimiento. Revista Teoría de la Educación, 2, 116-136.

\section{Para citar este artículo:}

SÁNCHEZ, C. \& ÁLVAREZ, M. J. (2013). Las competencias profesionales del tutor virtual ante las tecnologías emergentes de la sociedad del conocimiento. EDUTEC, Revista Electrónica de Tecnología Educativa, 44. Recuperado el dd/mm/aa de http://edutec.rediris.es/Revelec2/Revelec44/competencias profesionales tutor virtual.html 